\title{
Uso de Evidências Científicas na Avaliação da Incorporação de Tecnologias em SAÚdE NO SUS: UMA ANÁLISE PRELIMINAR ${ }^{1}$
}

Fabiola Sulpino Vieira²

Luciana Mendes Santos Servo ${ }^{3}$

Sergio Francisco Piola ${ }^{4}$

\section{INTRODUÇÃO}

O Sistema Único de Saúde (SUS) foi instituído em 1988 para assegurar o acesso da população a bens e a serviços de saúde de forma universal, igualitária e integral (Brasil, 1988). A integralidade implica a oferta de serviços de saúde em distintos níveis de complexidade, incluindo açóes de promoção, proteção e recuperação, a integração das práticas dos profissionais no cuidado aos pacientes e a implementaçáo de políticas para lidar com as diversas dimensóes inerentes ao sistema e aos cuidados de saúde (Mattos, 2009).

A avaliação da incorporação de tecnologias em saúde é parte do processo de formulação de políticas terapêuticas, que são componentes da Política Nacional de Assistência Farmacêutica. Segundo Souza (2003), o processo de formulação de uma política pública envolve a tradução dos propósitos dos governos em programas e açóes, com impactos sobre o mundo real. Logo, as políticas públicas constituem programas e ações governamentais, podendo-se compreender as políticas terapêuticas como políticas públicas que estabelecem as tecnologias empregadas para tratamento dos doentes e as condições para sua utilização, segundo as doenças que lhes acometem.

Elas podem ser implícitas, ou seja, não declaradas como tal, mas se caracterizam pelo propósito de orientar a oferta e a prescrição de terapias no sistema de saúde, a exemplo da Relação Nacional de Medicamentos Essenciais (Rename) (Brasil, 2019) e da Tabela SUS. ${ }^{5}$ Ou podem ser explícitas, quando claramente se apresentam com o propósito de orientar a prática dos profissionais de saúde, a exemplo dos Protocolos Clínicos e Diretrizes Terapêuticas (PCDT). ${ }^{6}$

Os medicamentos e os procedimentos técnicos sáo classificados ao lado dos equipamentos, sistemas organizacionais, informacionais, educacionais e de suporte, e dos programas e protocolos assistenciais como tecnologias em saúde (Brasil, 2010). Em um contexto de crescente desenvolvimento, oferta e custo dessas tecnologias, a existência de um mecanismo baseado em evidências, sistemático e reprodutível é fundamental para promover o acesso equitativo e sustentável aos cuidados de saúde para toda a população (WHO, 2002; Whitty, 2018).

No Brasil, a Comissão Nacional de Incorporação de Tecnologias no SUS (Conitec) é responsável pela avaliação das tecnologias em saúde, assessorando o secretário de Ciência, Tecnologia e Insumos

1. DOI: http://dx.doi.org/10.38116/bapi24art4

2. Especialista em políticas públicas e gestão governamental (EPPGG) na Diretoria de Estudos e Políticas Sociais (Disoc) do Ipea. E-mail:<fabiola.vieira@ipea.gov.br>.

3. Técnica de planejamento e pesquisa na Disoc/lpea.E-mail: <luciana.servo@ipea.gov.br>.

4. Pesquisador no projeto Acompanhamento e Análise da Política de Saúde na Disoc/lpea.E-mail: <sergiofpiola@gmail.com>.

5. A Tabela de Procedimentos, Medicamentos, Órteses, Próteses e Materiais Especiais do SUS (Tabela SUS) inclui procedimentos técnicos (cirúrgicos, psicoterapêuticos, entre outros) disponibilizados no sistema. Disponível em: <http://sigtap.datasus.gov.br/tabela-unificada/app/sec/inicio.jsp $>$.

6. Disponível em: <http://conitec.gov.br/protocolos-e-diretrizes>. 
Estratégicos do Ministério da Saúde na tomada de decisão sobre a sua incorporação ou desincorporação (Brasil, 2011). Com o apoio da Conitec e a participação de profissionais das distintas áreas técnicas do Ministério da Saúde, são formuladas as políticas terapêuticas de abrangência nacional, em um processo por si só desafiador, não apenas para o SUS, mas para todos os sistemas de saúde (Vieira, 2019).

Considerando este contexto, este artigo tem por objetivos apresentar os avanços do SUS no uso de evidências científicas na avaliação de tecnologias em saúde (ATS), segundo as orientações mais atuais nesse campo, e investigar, em caráter preliminar, se tais orientaçóes se concretizam no trabalho realizado pela Conitec.

Na seção 2, apresenta-se uma síntese da literatura científica que reporta os avanços do SUS nesta área desde 2011. E, na seção 3, discutem-se os resultados da análise preliminar de uma amostra de relatórios da Conitec, publicados em 2019, quanto ao nível das evidências utilizadas para fundamentar as recomendaçôes de uso das tecnologias com finalidades terapêuticas. Por fim, a síntese dos achados deste artigo é apresentada na seção 4, a título de considerações finais.

\section{USO DE EVIDÊNCIAS CIENTÍFICAS NA ATS NO SUS}

Conceitualmente, para o campo da saúde, as evidências estáo relacionadas a fatos utilizados para apoiar conclusões, que são conhecidos pela experiência ou observação. As evidências científicas são aquelas obtidas pela aplicação de métodos sistemáticos e replicáveis para sua produção, sendo a sua confiabilidade definida com base no método empregado para obtê-las (Lomas et al., 2005).

No SUS, a Conitec deve considerar as evidências científicas sobre a eficácia, a acurácia, a efetividade e a segurança do medicamento, produto ou procedimento avaliado, além de avaliaçôes econômicas sobre os benefícios e custos da tecnologia avaliada em relaçáo às já incorporadas ao sistema (Brasil, 2011).

A criação da Conitec em 2011 constituiu marco importante da ATS no país e promoveu avanços expressivos nesta área. Quanto à sua estrutura e seu funcionamento, a comissão apresenta mais similaridades do que diferenças em relação à estrutura e aos procedimentos adotados pelas agências de ATS da Austrália, Canadá e Reino Unido, que são consideradas referências nesta temática (Lima, Brito e Andrade, 2019).

Em menos de uma década, o Ministério da Saúde foi capaz de organizar os processos de trabalho da Conitec, alcançando resultados positivos, como maior qualidade e transparência na elaboração de suas guias de prática clínica em relação àquelas produzidas por sociedades de especialidades e pela Associação Médica Brasileira. Contudo, apesar dos avanços, ainda não obtiveram o mais alto nível possível para este tipo de documento (Molino et al., 2019).

Estudos recentes têm apontado falta de conformidade com a regulamentação interna da Conitec em termos do tipo e da qualidade das evidências. Do total de relatórios publicados em 2012 e 2016, em 87\% deles não foram observados os requisitos de um processo de ATS completo, havendo significativa diferença quanto às evidências utilizadas entre as demandas internas (do setor público) e as demandas externas (empresas produtoras/fornecedoras de tecnologias e sociedade civil), com menos rigor no caso das primeiras (Yuba, Novaes e Soárez, 2018). A probabilidade de aprovação das demandas internas foi quatro vezes maior em relação às externas para as solicitaçóes de incorporação de medicamentos entre 2012 e junho de 2016 (Caetano et al., 2017).

Há, portanto, questôes importantes a serem resolvidas quanto ao trabalho da comissão. Adiciona-se a elas a ausência de processo de reavaliação das tecnologias com revisão da sua 
indicação de uso ou recomendação para sua exclusão, ou seja, desinvestimento (Pereira, Barreto e Neves, 2019).

Outro problema de conformidade apontado é o da incorporação de medicamentos sem registro na Agência Nacional de Vigilância Sanitária (Anvisa) (Pedro et al., 2018). Esta é uma questão relevante, pois a ATS precisa considerar as finalidades das tecnologias. Na incorporaçáo de medicamentos para tratamento de doenças relacionadas à pobreza, a inexigibilidade do registro sanitário pode se justificar por seu valor terapêutico inconteste (Santana, Lupatini e Leite, 2017). A inexistência do registro pode demonstrar a falta de interesse das empresas na comercialização dos seus produtos no país. Neste caso, parece que a questão principal está mais relacionada à adequação da legislação às necessidades do SUS do que ao trabalho da Conitec. Em linhas gerais, a Conitec promoveu avanços significativos no processo de ATS no SUS, mas o trabalho da comissão demanda aperfeiçoamento.

\section{NIVEL DAS EVIDÊNCIAS EM RECOMENDAÇÕES DA CONITEC: UMA ANÁLISE PRELIMINAR}

No tocante à análise sobre o nível das evidências utilizadas para fundamentar suas recomendaçóes, do total de 77 relatórios publicados em 2019, 68 abordam tecnologias com finalidades terapêuticas. Recomendam a incorporação ou a exclusão de medicamentos e procedimentos técnicos ou a aprovação de PCDT e de outras diretrizes terapêuticas. Neste estudo, trabalhou-se com uma amostra de 20\% $(\mathrm{n}=14)$ desses relatórios, selecionados por meio de amostragem aleatória estratificada. $\mathrm{O}$ quadro 1 apresenta uma síntese dos achados sobre o nível das evidências utilizadas, considerando-se o delineamento dos estudos citados, conforme descrito por Fletcher e Fletcher (2006), e a classificação dos níveis de evidência quanto à eficácia e efetividade das intervenções, de acordo com os procedimentos iniciais da metodologia GRADE (Grading of Recommendations Assessment, Development and Evaluation) para avaliação da qualidade da evidência (Brasil, 2014).

Em linhas gerais, nessa análise preliminar, observa-se que, para a maioria dos relatórios analisados, o nível das evidências consideradas pode ser classificado como moderado ou alto, implicando confiança mediana a forte no efeito estimado, para a maioria das intervençóes recomendadas pela Conitec. Todavia, há uma parcela não desprezível delas que foi considerada com nível baixo ou muito baixo das evidências científicas, a exemplo das intervençóes para tratamento da intoxicação por glifosato, da indicação de nusinersena para atrofia muscular espinhal (AME) 5q tipo 1, do transplante de células-tronco hematopoiética na mucopolissacaridose tipo IV A e de dicloridrato de sapropterina no tratamento da fenilcetonúria.

Os níveis baixo e muito baixo das evidências para intervençóes utilizadas no tratamento de intoxicações agudas e de doenças raras podem ser justificados pela impossibilidade ou pela dificuldade de realização de ensaios clínicos. No primeiro caso, por questóes óbvias, e, no segundo, porque é pequeno o número de pacientes que poderiam ser recrutados para a realização dessas pesquisas. Com isso, as evidências acabam sendo oriundas de estudos observacionais, como de caso e controle e relatos de caso, os quais proveem limitada confiança no efeito da intervenção.

No caso das doenças raras, os vazios terapêuticos, ou seja, a inexistência de alternativas para tratamento, costuma fazer com que as agências de ATS admitam evidências menos robustas sobre os efeitos das intervenções. Mas este não foi o caso da Conitec ao indicar a realização do transplante de células-tronco hematopoiética, o uso de nusinersena e de sapropterina, haja vista a disponibilidade no SUS de alternativas terapêuticas com nível mais elevado de evidências para as indicaçốes mencionadas (quadro 1). 


\section{QUADRO 1}

\section{Níveis de evidências preliminares ${ }^{1}$ para as intervenções recomendadas pela Conitec, segundo o sistema GRADE, para a amostra de relatórios analisada}

\begin{tabular}{|c|c|c|c|c|c|c|}
\hline \multirow[b]{2}{*}{ Tecnologias, relatórios e intervenções } & \multirow[b]{2}{*}{ Recomendação } & \multicolumn{4}{|c|}{ Tipos de estudo sobre tratamento (quantidades) } & \multirow[b]{2}{*}{$\begin{array}{l}\text { Níveis de } \\
\text { evidência } \\
\text { (Grupo GRADE } \\
\text { preliminar) }\end{array}$} \\
\hline & & $\begin{array}{l}\text { Revisões } \\
\text { sistemáticas } \\
\text { com e sem } \\
\text { metanálise }\end{array}$ & $\begin{array}{l}\text { Ensaios } \\
\text { clínicos }\end{array}$ & $\begin{array}{l}\text { Estudos } \\
\text { observacionais } \\
\text { (coorte, caso } \\
\text { e controle) }\end{array}$ & $\begin{array}{l}\text { Outros (séries de } \\
\text { casos, relatos de } \\
\text { casos, diretrizes, } \\
\text { entre outros) }\end{array}$ & \\
\hline \multicolumn{7}{|c|}{ PCDTs e diretrizes terapêuticas } \\
\hline $\begin{array}{l}\text { Relatório no } 494 \text { - Protocolo clínico e diretrizes } \\
\text { terapêuticas da mucopolissacaridose tipo IV A } \\
\text { (síndrome de Morquio A) }\end{array}$ & Aprovar PCDT & & & & & \\
\hline $\begin{array}{l}\text { Transplante de células-tronco } \\
\text { hematopoiética }\end{array}$ & & - & - & - & 3 & Muito baixo \\
\hline Terapia de reposição enzimática & & - & 1 & - & 3 & Moderado \\
\hline $\begin{array}{l}\text { Relatório n } 492 \text { - PCDT - Atrofia Muscular } \\
\text { Espinhal (AME) } 5 q \text { Tipo } 1\end{array}$ & Aprovar PCDT & & & & & \\
\hline Nusinersena & & - & 1 & & 1 & Baixo \\
\hline $\begin{array}{l}\text { Terapia não farmacológica (cuidados } \\
\text { nutricionais, ortopédicos e respiratórios) }\end{array}$ & & - & - & - & 6 & Muito baixo \\
\hline Relatório no 465 - PCDT Fenilcetonúria & Aprovar PCDT & & & & & \\
\hline Dieta restritiva em fenilalanina & & - & 2 & - & 10 & Moderado \\
\hline Fórmula de aminoácido isenta de fenilalanina & & 1 & 1 & - & 3 & Moderado \\
\hline Dicloridrato de sapropterina & & - & - & - & 2 & Muito baixo \\
\hline Relatório no 455 - PCDT Esclerose múltipla & Aprovar PCDT & & & & & \\
\hline Acetato de glatirâmer & & 1 & 5 & - & 1 & Moderado a alto \\
\hline $\begin{array}{l}\text { Relatório no } 425 \text { - Diretrizes brasileiras para } \\
\text { diagnóstico e tratamento das intoxicações por } \\
\text { agrotóxicos - capítulo } 3\end{array}$ & Aprovar diretrizes & & & & & \\
\hline Descontaminação da pele e mucosas & & - & - & - & 3 & Muito baixo \\
\hline $\begin{array}{l}\text { Hemoperfusão Direta ou Hemodiafiltração } \\
\text { Venovenosa Contínua }\end{array}$ & & - & - & - & 3 & Muito baixo \\
\hline Hemodiálise & & - & - & - & 2 & Muito baixo \\
\hline $\begin{array}{l}\text { Terapia adjuvante (administração parenteral } \\
\text { de emulsão lipídica) }\end{array}$ & & - & 1 & - & 2 & Baixo \\
\hline $\begin{array}{l}\text { Relatório no } 401 \text { - Diretrizes diagnósticas e } \\
\text { terapêuticas de neoplasia maligna epitelial } \\
\text { de ovário }\end{array}$ & Aprovar diretrizes & & & & & \\
\hline Cirurgia & & 3 & 3 & 3 & 1 & Alto \\
\hline Quimioterapia neoadjuvante & & 6 & 2 & 3 & - & Alto \\
\hline Quimioterapia adjuvante & & 2 & - & - & - & Moderado a alto \\
\hline Quimioterapia intraperitoneal & & 1 & - & 2 & 1 & Moderado \\
\hline Tratamento da recidiva tumoral & & 4 & 1 & & 2 & Alto \\
\hline $\begin{array}{l}\text { Tratamento da doença refratária a } \\
\text { compostos de platina }\end{array}$ & & 1 & 3 & 1 & - & Moderado a alto \\
\hline Terapia endócrina & & 1 & 1 & 2 & - & Moderado a alto \\
\hline Tratamento de manutenção & & 2 & - & - & - & Moderado a alto \\
\hline
\end{tabular}


(Continuação)

\begin{tabular}{|c|c|c|c|c|c|c|}
\hline \multirow[b]{2}{*}{ Tecnologias, relatórios e intervenções } & \multirow[b]{2}{*}{ Recomendação } & \multicolumn{4}{|c|}{ Tipos de estudo sobre tratamento (quantidades) } & \multirow[b]{2}{*}{$\begin{array}{l}\text { Níveis de } \\
\text { evidência } \\
\text { (Grupo GRADE } \\
\text { preliminar) }\end{array}$} \\
\hline & & $\begin{array}{l}\text { Revisões } \\
\text { sistemáticas } \\
\text { com e sem } \\
\text { metanálise }\end{array}$ & $\begin{array}{l}\text { Ensaios } \\
\text { clínicos }\end{array}$ & $\begin{array}{l}\text { Estudos } \\
\text { observacionais } \\
\text { (coorte, caso } \\
\text { e controle) }\end{array}$ & $\begin{array}{l}\text { Outros (séries de } \\
\text { casos, relatos de } \\
\text { casos, diretrizes, } \\
\text { entre outros) }\end{array}$ & \\
\hline \multicolumn{7}{|c|}{ Medicamentos } \\
\hline $\begin{array}{l}\text { Relatório no } 486 \text { - Certolizumabe pegol (CZP) } \\
\text { para o tratamento da artrite psoríaca (PzA) }\end{array}$ & $\begin{array}{l}\text { Incorporar } \\
\text { ao SUS }\end{array}$ & 1 & 7 & 1 & - & $\begin{array}{l}\text { Moderado } \\
\text { a baixo }\end{array}$ \\
\hline $\begin{array}{l}\text { Relatório no } 484 \text { - Secuquinumabe como } \\
\text { primeira etapa de terapia biológica para o } \\
\text { tratamento da espondilite anquilosante ativa } \\
\text { em pacientes adultos }\end{array}$ & $\begin{array}{c}\text { Não incorporar } \\
\text { ao SUS }\end{array}$ & 5 & - & - & - & Alto \\
\hline $\begin{array}{l}\text { Relatório no } 454 \text {-Sacubitril/valsartana para } \\
\text { o tratamento de pacientes adultos com } \\
\text { insuficiência cardíaca crônica sintomática } \\
\text { (NYHA classe II-IV) com fração de } \\
\text { ejeção reduzida }\end{array}$ & $\begin{array}{l}\text { Incorporar } \\
\text { ao SUS }\end{array}$ & 2 & 1 & 2 & - & Moderado \\
\hline $\begin{array}{l}\text { Relatório no } 464 \text { - Abiraterona para câncer de } \\
\text { próstata metastático resistente à castração em } \\
\text { pacientes com uso prévio de quimioterapia }\end{array}$ & $\begin{array}{l}\text { Incorporar } \\
\text { ao SUS }\end{array}$ & 1 & 2 & - & - & Moderado a alto \\
\hline $\begin{array}{l}\text { Relatório no } 458 \text { - Tetraciclina } 500 \text { mg para o } \\
\text { tratamento de hidradenite supurativa leve }\end{array}$ & $\begin{array}{l}\text { Incorporar } \\
\text { ao SUS }\end{array}$ & - & 1 & 1 & 4 & Moderado \\
\hline $\begin{array}{l}\text { Relatório no } 440 \text { - Insulinas análogas de ação } \\
\text { prolongada para o tratamento de diabetes } \\
\text { mellitus tipo I }\end{array}$ & $\begin{array}{l}\text { Incorporar } \\
\text { ao SUS }\end{array}$ & 7 & - & 5 & 2 & Moderado a alto \\
\hline $\begin{array}{l}\text { Relatório no } 248 \text { - Simeprevir para o } \\
\text { tratamento da hepatite C }\end{array}$ & Excluir & - & - & - & - & $\begin{array}{c}\text { Sem } \\
\text { classificação² }\end{array}$ \\
\hline \multicolumn{7}{|c|}{ Procedimento técnico } \\
\hline $\begin{array}{l}\text { Relatório no } 364 \text {-Transplante de fígado para } \\
\text { insuficiência hepática hiperaguda relacionada } \\
\text { à febre amarela }\end{array}$ & $\begin{array}{l}\text { Incorporar } \\
\text { ao SUS }\end{array}$ & - & - & - & - & $\begin{array}{c}\text { Sem } \\
\text { classificação² }\end{array}$ \\
\hline
\end{tabular}

Elaboração dos autores.

Notas: ${ }^{1} \mathrm{~A}$ classificação GRADE foi realizada considerando apenas a quantidade de estudos por tipo. Não foi feita uma avaliação GRADE completa, contemplando a análise da qualidade de cada estudo.

${ }^{2}$ Nenhum estudo foi citado no relatório.

Recentemente, o processo de incorporação do medicamento nusinersena, o mais caro já incorporado ao SUS, gerou preocupaçôes quanto à reversão do curso da institucionalização da ATS no sistema, até então caracterizado pela busca da transparência e da accountability. Os autores de um estudo sobre este caso concluem que a recomendação para a incorporação se deu por decisão política, uma vez que as evidências sobre a eficácia, efetividade e custo-efetividade do medicamento não apoiam tal recomendaçáo (Caetano, Hauegen e Osório-de-Castro, 2019). Na análise do PCDT que trata dessa doença, realizada no presente artigo, observou-se também um relatório com construção menos elaborada, ainda que náo tenha sido analisado o relatório de incorporação do medicamento. ${ }^{7}$

Quando se comparam os PCDT da AME 5q tipo 1 e da esclerose múltipla, observa-se significativa diferença na organização dos relatórios e na qualidade das evidências apresentadas. No primeiro relatório, apenas um ensaio clínico randomizado é apresentado como evidência para tratamento com

7. No caso do nusinersena, no relatório sob análise é citado outro relativo à recomendação de incorporação desse medicamento. Neste último, há referências a outros ensaios clínicos randomizados. Entretanto, pela lógica de organização dos protocolos, eles deveriam apresentar todas as evidências que o embasaram. 
nusinersena. No segundo, além de se apresentarem evidências para todos os medicamentos de cada linha de tratamento, especificamente no caso do glatiramer, são apresentados cinco ensaios clínicos e uma revisão sistemática. Nesta primeira análise, o segundo relatório parece ser bem detalhado e exaustivo na avaliação das evidências em relação ao primeiro.

Também foram publicados relatórios sem embasamento das recomendaçóes por evidências científicas. Um deles trata da exclusão do medicamento simeprevir para o tratamento da hepatite C, porque a empresa produtora deixou de oferecer o produto no mercado nacional por motivos comerciais, situação que dispensa a apresentação de evidências científicas. Contudo, em outro relatório, recomenda-se a realização de transplante de fígado para insuficiência hepática hiperaguda relacionada à febre amarela, com base apenas em dados do Sistema Nacional de Transplantes.

Mudanças de posicionamento da comissão após a realização da consulta pública, sem que novas evidências científicas tenham sido apresentadas e as fundamentassem, também foram observadas nos relatórios. Uma primeira análise do relatório de avaliação da incorporação do CZP para o tratamento da PzA poderia indicar que as evidências teriam uma boa avaliação pelo GRADE, visto que incluem muitos ensaios clínicos randomizados. Todavia, a avaliaçáo da própria Conitec foi de que as evidências apresentadas pelo demandante eram frágeis do ponto de vista metodológico. Nesse momento, a recomendação era pela não incorporação. Após a consulta pública, a comissão argumentou que os dados de comparaçáo indireta mostravam eficácia equivalente do CZP à da alternativa incorporada (secuquinumabe $300 \mathrm{mg}$ ), mas com economia do primeiro em comparação com o segundo. Assim, a recomendação foi de incorporá-lo ao SUS.

Algo semelhante ocorreu com a avaliação das insulinas análogas. A análise das evidências gerou uma recomendação inicial de não incorporação. Contudo, após as consultas públicas, e considerando outros fatores, como as açóes judiciais, a Conitec passou a recomendar sua incorporaçáo ao sistema. Também o sacubitril/valsartana, medicamento para pacientes com insuficiência cardíaca crônica sintomática com fração de ejeção reduzida, teve sua incorporação aprovada depois da consulta pública, mesmo com decisão inicial contrária da comissão, por considerar os benefícios clínicos não condizentes com o preço praticado no Brasil.

O processo de ATS pela Conitec prevê a utilização de evidências econômicas provenientes de análises de custo-efetividade ${ }^{8}$ e de impacto orçamentário. Constatou-se, neste trabalho, que essas evidências econômicas não são determinantes na decisão da comissão, mesmo diante de benefícios clínicos ainda baseados em poucos estudos. Esse é o caso do acetato de abiraterona, usado no tratamento do adenocarcinoma de próstata metastático resistente à castraçáo, em que, apesar de o relatório demonstrar impacto orçamentário relevante e relação custo-efetividade desfavorável, a comissão se posicionou pela incorporação.

Quanto à pressão exercida pela judicialização da saúde sobre a Conitec, os autores de um estudo, no qual foram analisadas atas de reuniôes e conteúdo de entrevistas feitas com membros dessa comissão, concluíram que as açóes judiciais não influenciaram diretamente o seu processo de ATS (Souza, Souza e Lisboa, 2018). Todavia, ao menos no processo recente de avaliação das insulinas análogas, as açóes judiciais são citadas no relatório como uma das justificativas para sua incorporação.

8. São avaliações em que os custos e as consequências de programas de saúde ou de tratamentos são examinados (Drummond et al., 2005). 


\section{CONSIDERAÇÕES FINAIS}

O processo de avaliação da incorporação de tecnologias com base em evidências é uma prática adotada por vários países do mundo. Uma das agências governamentais conhecidas é o National Institute for Health Care and Excellence (NICE), criado para, entre outros objetivos, apoiar a tomada de decisão sobre a incorporação de tecnologias em saúde no sistema nacional de saúde do Reino Unido. A Conitec se espelha nessa e em outras experiências para apoiar a tomada de decisão no SUS. Assim, considerando-se que as evidências científicas devem ser importantes balizadores das decisões da Conitec, justifica-se a escolha desse objeto para uma análise preliminar do uso de evidências nas políticas públicas de saúde.

Com base nos resultados de estudos recentemente publicados sobre o processo de trabalho da Conitec e na análise preliminar sobre o nível das evidências consideradas pela comissão para a realização de recomendaçóes sobre intervençóes com finalidades terapêuticas, conclui-se que houve avanços importantes quanto à ATS no SUS nos últimos anos. Entretanto, ainda se observam desconformidades significativas quanto às práticas recomendadas para um processo de ATS robusto e confiável, conforme sinaliza esta análise realizada sobre o nível das evidências.

Como neste trabalho não se avançou na avaliação dos fatores que permitem reduzir ou elevar o nível das evidências de acordo com o sistema GRADE, tais como limitaçóes metodológicas das pesquisas, inconsistência, imprecisão e viés de publicação, a análise ora apresentada tem caráter preliminar. Estudos mais detalhados precisam ser feitos para investigar esses e outros fatores que impactam o nível das evidências consideradas e o processo de ATS ora em curso no SUS, como, por exemplo, a possibilidade de captura, por agentes do complexo econômico-industrial da saúde, de membros da comissão ou de tomadores de decisão no Ministério da Saúde, os quais, neste último caso, têm a palavra final sobre a incorporação da tecnologia avaliada.

\section{REFERÊNCIAS}

BRASIL. Constituição da República Federativa do Brasil de 1988. Diário Oficial da Uniáo, Brasília, 5 out. 1988.

- Ministério da Saúde. Política Nacional de Gestão de Tecnologias em Saúde. Brasília: Ministério da Saúde, 2010.

. Lei no 12.401, de 28 de abril de 2011. Altera a Lei no 8.080, de 19 de setembro de 1990, para dispor sobre a assistência terapêutica e a incorporação de tecnologia em saúde no âmbito do Sistema Único de Saúde - SUS. Diário Oficial da União, Brasília, 2011.

Sistema GRADE: manual de graduação da qualidade da evidência e força de recomendação para tomada de decisão em saúde. Brasília: Ministério da Saúde, 2014.

Relação Nacional de Medicamentos Essenciais 2020. Brasília: Ministério da Saúde, 2019.

CAETANO, R. et al. Incorporação de novos medicamentos pela Comissão Nacional de Incorporação de Tecnologias do SUS, 2012 a junho de 2016. Ciência e Saúde Coletiva, Rio de Janeiro, v. 22, n. 8, p. 2513-2525, 2017. 
CAETANO, R.; HAUEGEN, R. C.; OSORIO-DE-CASTRO, C. G. S. A incorporação do nusinersena no Sistema Único de Saúde: uma reflexão crítica sobre a institucionalização da avaliação de tecnologias em saúde no Brasil. Cadernos de Saúde Pública, Rio de Janeiro, v. 35, n. 8, e00099619, 2019.

DRUMMOND, M. F. et al. Methods for the economic evaluation of health care programmes. New York: Oxford University Press, 2005.

FLETCHER, R. H.; FLETCHER, S. W. Epidemiologia clínica: elementos essenciais. 4. ed. Porto Alegre: Artmed, 2006.

LIMA, S. G. G.; BRITO, C.; ANDRADE, C. J. C. O processo de incorporação de tecnologias em saúde no Brasil em uma perspectiva internacional. Ciência e Saúde Coletiva, Rio de Janeiro, v. 24, n. 5, p. 1709-1722, 2019.

LOMAS, J. et al. Conceptualizing and combining evidence for health system guidance. [s.l.]: Canadian Health Services Research Foundation, 2005.

MATTOS, R. A. Os sentidos da integralidade: algumas reflexóes acerca de valores que merecem ser defendidos. In: PINHEIRO, R.; MATTOS, R. A. (Org.). Os sentidos da integralidade na atençáo e no cuidado à saúde. 8. ed. Rio de Janeiro: Abrasco, 2009.

MOLINO, C. G. R. C. et al. Comparison of the methodological quality and transparency of Brazilian practice guidelines. Ciência e Saúde Coletiva, Rio de Janeiro, v. 24, n. 10, p. 3947-3956, 2019.

PEDRO, E. M. et al. Incorporação de medicamentos sem registro sanitário no SUS: um estudo das recomendaçóes da Comissão Nacional de Incorporação de Tecnologias no período 2012-2016. Revista Visa em Debate, Rio de Janeiro, v. 6, n. 3, p. 12-21, 2018.

PEREIRA, V. C.; BARRETO, J. O. M.; NEVES, F. A. R. Health technology reassessment in the Brazilian public health system: analysis of the current status. PLoS ONE, San Francisco, v. 14, n. 7, e0220131, 2019.

SANTANA, R. S.; LUPATINI, E. O.; LEITE, S. N. Registro e incorporação de tecnologias no SUS: barreiras de acesso a medicamentos para doenças da pobreza? Ciência e Saúde Coletiva, Rio de Janeiro, v. 22, n. 5, p. 1417-1428, 2017.

SOUZA, C. Políticas públicas: questôes temáticas e de pesquisa. Caderno CRH, Salvador, v. 16, n. 39, p. 11-24, 2003.

SOUZA, K. A. O.; SOUZA, L. E. P. F.; LISBOA, E. S. Açôes judiciais e incorporação de medicamentos ao SUS: a atuação da Conitec. Saúde em Debate, Rio de Janeiro, v. 42, n. 119, p. 837-848, 2018.

VIEIRA, F. S. Desafios do Estado quanto à incorporaçáo de medicamentos no Sistema Único de Saúde. Brasília: Ipea, 2019. (Texto para Discussão, n. 2.500).

WHITTY, J. A. Health technology assessment. In: BABAR, Z. Equitable access to high-cost pharmaceuticals. London: Elsevier, 2018.

WHO - WORLD HEALTH ORGANIZATION. Promoting rational use of medicines: core components. Geneva: WHO, 2002.

YUBA, T. Y.; NOVAES, H. M. D.; SOÁREZ, P. C. Challenges to decision-making processes in the national HTA agency in Brazil: operational procedures, evidence use and recommendations. Health Research Policy and Systems, London, v. 16, n. 1, p. 40, 2018. 